\title{
Joint Resource Allocation for Adaptive Fuzzy Logic Based Coordinated Multi-Cell NOMA Systems
}

\author{
Haiyong Zeng*, Xu Zhu*†, Yufei Jiang* and Zhongxiang Wei ${ }^{\star}$ \\ * School of Electronic and Information Engineering, Harbin Institute of Technology, Shenzhen, China \\ $\dagger$ School of Electrical Engineering and Electronics, University of Liverpool, Liverpool, UK \\ * School of Electrical Engineering and Electronics, University College London, London, UK \\ Emails: zenghaiyong@ @stu.hit.edu.cn, xuzhu@liverpool.ac.uk, jiangyufei@hit.edu.cn, zhongxiang.wei@ucl.ac.uk
}

\begin{abstract}
We investigate a downlink multi-cell non-orthogonal multiple access (NOMA) system with coordinated base stations (BSs) and propose a joint resource allocation (RA) scheme alongside adaptive user association to green the system. To the best of our knowledge, this is the first work to investigate joint allocation of subchannels and power for coordinated NOMA systems, while the previous work on RA for coordinated orthogonal multiple access (OMA) systems is not applicable. A serving channel gain based joint RA (SCG-JRA) algorithm is proposed, based on the theoretical proof that the total transmission power is mono-decreasing with respect to the SCGs of non-coordinated users. As for user association, an adaptive fuzzy logic (FL) based multi-criterion approach is proposed to achieve higher robustness against the combined effect of shadowing, fading and inter-cell interference, compared to the previous single-criterion based approaches. Numerical results show that the proposed SCG-JRA with adaptive FL based user association significantly outperforms the previous RA schemes assisted by single-criterion user association, in terms of energy efficiency (EE) and total transmission power, enabling a greener system.
\end{abstract}

\section{INTRODUCTION}

Non-orthogonal multiple access (NOMA) [1] [2], which allows the same frequency to be shared among multiple users simultaneously, has been envisioned as a promising technology for the fifth generation (5G) system. By utilizing power-domain multi-user multiplexing and successive interference cancellation (SIC), NOMA allows receivers to decode and demodulate the superposition of the encoded signals [2]. In a more practical scenario, i.e., multi-cell NOMA, the users close to the edge of cells generally suffer from poor channel condition and strong inter-cell interference. In order to achieve the target quality of service (QoS), more transmission power is required for those users, which is not power efficient and causes stronger multicell interference [3] [4]. To alleviate this, coordination between base stations (BSs) can be exploited to jointly transmit signals to the target users [5]-[8]. It has been demonstrated in [8] that the system with multiple coordinated BSs can benefit from the potential distributed space diversity gains, and the coordinated NOMA system provides better performance than

This work was supported in part by the Science and Technology Innovation Commission of Shenzhen under Project No. JCYJ20170307151258279, the Natural Science Foundation of Guangdong Province under grant No. 2018A030313298 and No. 2018A030313344, and the "Liverpool 5G" Project, Department for Digital, Culture, Media and Sport (DCMS), UK. the non-coordinated NOMA and the coordinated orthogonal multiple access (OMA) system, respectively.

It is worthy noting that, the resource allocation (RA) in terms of subchannel assignment and power allocation plays a critical role in achieving green communication for coordinated NOMA systems. Since multiple users can share the same subchannel in coordinated NOMA, the previous work on RA proposed for coordinated OMA systems [3] [4] cannot be utilized directly for coordinated NOMA systems. The authors in [9] proposed an energy-efficiency (EE)-oriented single channel power allocation algorithm for coordinated NOMA systems. In [10], an opportunistic coordinated NOMA scheme was proposed to enhance the performance. However, the work in [9] and [10] has only focused on power allocation for coordinated NOMA, assuming a single-channel model, which is not practical for current standards using multiple subchannels.

In addition, the performance of coordinated NOMA is largely influenced by user association [9]-[12]. Current user association approaches are based on a single criterion, and can be mainly categorized into two types: a) distance based approaches [9] [11]; b) channel gain based approaches [10] [12]. However, the aforementioned distance based approaches are sensitive to fading and shadowing, while the performance of the channel gain based approaches can be severely affected by inter-cell interference, leading to poor system performance. To this end, multiple criteria are necessary for user association to enhance the robustness. As mentioned in [13], fuzzy logic (FL) is an effective artificial intelligence (AI) approach to make a dynamic decision based on multiple input parameters. In our previous work [7], FL based user association was proposed to balance between multiple criteria, which outperforms singlecriterion based approaches. However, its fuzzy membership function is predetermined and cannot be tuned adaptively to the change of network environment, which results in performance degradation, especially when users are under deep shadowing and fading. Hence, adaptive FL based user association in coordinated NOMA is preferred. Moreover, the RA scheme in [7] merely accounts for user association and lacks joint allocation of subchannels and power. While the joint RA for coordinated NOMA systems still remains an open challenge.

In this paper, we consider a downlink multi-cell NOMA system with coordinated $\mathrm{BSs}$ and investigate its joint RA 
alongside adaptive user association to green the system. The contributions are summarized as follows:

1) To the best of our knowledge, this is the first reported work to investigate joint allocation of multiple subchannels and power for coordinated NOMA systems, alongside user association. While the RA methods dedicated for coordinated OMA systems [3] [4] are not applicable to coordinated NOMA. Also, the previous work for coordinated NOMA either assumed a single-subchannel model [9] [10] or lacks joint RA [7].

2) We propose an adaptive FL based user association scheme, where multiple criteria, i.e., distance, channel gain and reference signal received power (RSRP) are considered. The fuzzy membership function is tuned adaptively to the change of shadowing standard deviation so that the FL based scheme can be adaptive to different network environment and enlarge the difference of suitability of users under deep shadowing and fading, which was not considered in [7]. The proposed adaptive FL based scheme is more robust against fading, shadowing and inter-cell interference than the method in [7] and the previous single-criterion based schemes [9] [10].

3) An intensive analysis is provided to theoretically prove that the total transmission power of the system is monodecreasing with respect to the serving channel gains (SCGs) of the non-coordinated users. In light of that, we propose an SCG based joint RA (SCG-JRA) algorithm for allocation of subchannels and power across users, with an optimal closed-form power allocation result. The proposed SCG-JRA alongside adaptive FL based user association significantly outperforms the previous RA schemes with single-criterion user association [9] [10] and the RA scheme in [7], enabling a greener system with less transmission power.

\section{System Model And Problem Formulation}

\section{A. System Model}

We consider a multi-cell downlink NOMA system with $B$ BSs and $L$ users. Assume that the overall bandwidth of the coordinated NOMA system $W$ is divided uniformly into $M$ subchannels (SCs), and each BS transmits messages to its serving users through these SCs. As depicted in Fig. 1, for user association, users in the system are chosen to work in two modes: non-coordinated mode and coordinated mode. Denote $L_{C}$ as the number of the coordinated users in the system. Let $x_{l, m} \in\{0,1\}$, in which $x_{l, m}=1$ indicates that user $l$ is allocated to SC $m$. Define $U_{m, b}$ as the set of users served by $\mathrm{BS} b(b=1, \ldots, B)$ on $\mathrm{SC} m(m=1, \ldots, M)$ and $B_{l}$ as the set of distributed coordinated BS(s) serving for user $l$. Let $p_{l, m}$ denote the transmission power allocated to user $l$ on SC $m$. Then, the received signal at user $l$ on SC $m$ is

$$
\begin{aligned}
y_{l, m}= & \sum_{b \in B_{l}} h_{b, l, m}\left(\sqrt{p_{l, m}} s_{l, m}+\sum_{i \in U_{m, b}, i \neq l} \sqrt{p_{i, m}} s_{i, m}\right) \\
& +\sum_{b \in\left\{B / B_{l}\right\}} h_{b, l, m} \sum_{i \in U_{m, b}} \sqrt{p_{i, m}} s_{i, m}+\omega_{l, m} .
\end{aligned}
$$

where $s_{l, m}$ and $s_{i, m}$ are the data symbols with unit energy and $h_{b, l, m}$ denotes the Rayleigh fading channel state information

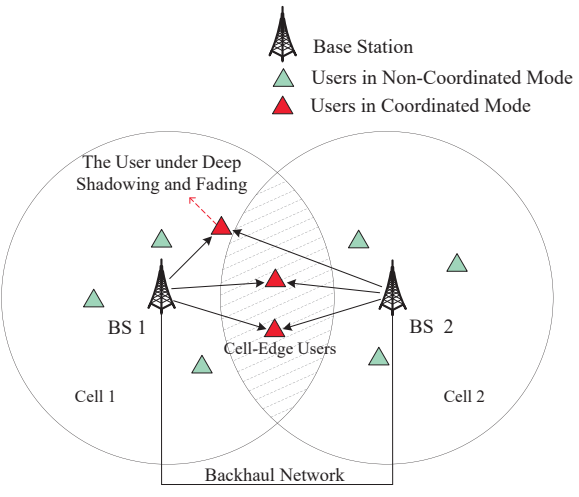

Fig. 1. System model of multi-cell coordinated NOMA systems.

(CSI) from BS $b$ to user $l$ on SC $m$, which is affected by shadowing and path loss. $\omega_{l, m}$ stands for the additional white complex Gaussian noise $\omega_{l, m} \sim \mathbb{C} M\left(0, \sigma_{m}^{2}\right)$.

At the receiver, SIC process is implemented to decode the received signals. Define $H_{b, l, m}=\left|h_{b, l, m}\right|^{2}$ as the channel gain of user $l$. According to NOMA protocol, the SIC process on SC $m$ is the increasing order of channel gains of multiplexed users [2]. After SIC, the received signal-to-interference-plusnoise ratio for user $l$ on $\mathrm{SC} m$ is given by

$$
\gamma_{l, m}=\frac{p_{l, m} \sum_{b \in B_{l}} H_{b, l, m}}{\sigma_{m}^{2}+\phi_{l, m}+\psi_{l, m}},
$$

where $\psi_{l, m}$ stands for the inter-cell interference gain and $\phi_{l, m}$ is the intra-cell interference gain, respectively.

As mentioned above, the users can be selected to work in the coordinated or non-coordinated mode. Assume the CSI of the coordinated users is available to the coordinated BSs, and the messages sent to user $l$ by $B_{l}$ share the same transmit power [9]. On one hand, if user $l$ is in the coordinated mode, due to the coordination of BSs [7]-[9], the inter-cell interference of user $l$ from the coordinated BSs $B_{l}$ is 0 , while the intra-cell gain for user $l$ can be obtained as

$$
\phi_{l, m}=\sum_{b \in B_{l}} H_{b, l, m} \sum_{i \in\left\{U_{m, b}, l\right.} p_{i, m},
$$

where $U_{m, b, l}=\left\{\tilde{l} \mid H_{b, \tilde{l}, m}>H_{b, l, m}, \tilde{l} \in U_{m, b}\right\}$. Therefore, the achievable data rate (in bps/Hz) of user $l$ on SC $m$ is

$$
r_{l, m}=c_{l} \log _{2}\left(1+\frac{p_{l, m} \sum_{b \in B_{l}} H_{b, l, m}}{\sigma_{m}^{2}+\sum_{b \in B_{l}} H_{b, l, m} \sum_{i \in\left\{U_{m, b}, l\right.} p_{i, m}}\right),
$$

where $c_{l} \in\{0,1\}, c_{l}=1$ means user $l$ is in the coordinated mode, otherwise user $l$ is in the non-coordinated mode.

On the other hand, if user $l$ is in the non-coordinated mode (i.e., $c_{l}=0$ ), the intra-cell interference gain $\phi_{l, m}$ and inter-cell interference gain $\psi_{l, m}$ can be expressed as

$$
\phi_{l, m}=H_{B_{l}, l, m} \sum_{i \in\left\{\tilde{U}_{m, B_{l}, l}\right\}} p_{i, m}
$$




$$
\psi_{l, m}=\sum_{b \in\left\{B \backslash B_{l}\right\}} H_{b, l, m} \sum_{j \in\left\{U_{m, b}\right\}} p_{j, m}
$$

where $\tilde{U}_{m, B_{l}, l}=\left\{\tilde{l} \mid H_{B_{l}, \tilde{l}, m}>H_{B_{l}, l, m}, \tilde{l} \in\left\{U_{m, B_{l}}\right\}\right\}$. Following [9], we assume that there is one coordinated user served by $B_{l}$ on each SC.

The achievable data rate of non-coordinated user $l$ is

$r_{l, m}=\left(1-c_{l}\right) \times$

$\log _{2}\left(1+\frac{p_{l, m} H_{B_{l}, l, m}}{\sigma_{m}^{2}+H_{B_{l}, l, m} \sum_{i \in\left\{\tilde{U}_{m, B_{l}, l}\right\}} p_{i, m}+\sum_{b \in\left\{B \backslash B_{l}\right\}} H_{b, l, m} \sum_{j \in\left\{U_{m, b}\right\}} p_{j, m}}\right)$.

Remark 1: For multi-cell coordinated NOMA systems, the users located in the edge of cells can suffer from poor channel conditions and strong inter-cell interference. Also, the users close to the serving BS who suffer severe fading and shadowing, have poor channel conditions. To improve performance, these users are chosen in the coordinated mode.

\section{B. Problem Formulation}

Let $\mathbf{X}=\left[x_{l, m}\right]_{L \times M}$ denote the subchannel assignment matrix, $\mathbf{C}=\left[c_{l}\right]_{L \times 1}$ be the user association matrix, and $\mathbf{P}=\left[p_{l, m}\right]_{L \times M}$ be the power allocation matrix.

Then the total transmission power $P_{t}$ is

$$
P_{t}=\sum_{m=1}^{M} \sum_{b=1}^{B} \sum_{l \in U_{m, b}} p_{m, l} .
$$

Therefore, the optimization problem for the downlink multicell coordinated NOMA system can be formulated as

$$
\underset{\mathbf{P}, \mathbf{X}, \mathbf{C}}{\arg \min } \eta(\mathbf{P}, \mathbf{X}, \mathbf{C})=P_{t},
$$

subject to $(\mathrm{C} 1): x_{l, m} \in\{0,1\},(\mathrm{C} 2): c_{l} \in\{0,1\} ;(\mathrm{C} 3)$ : $\sum_{l \in U_{m, b}} x_{l, m}=G$; (C4): $r_{l, m} \geq r_{\min }$; (C5): $p_{m, l} \geq 0$, where $r_{\min }$ denotes the QoS requirement, and $G$ is the maximum number of allocated users sharing the same SC. As mentioned in [2], at receiver side, the implementation complexity of SIC increases with $G$. In order to make a trade-off between performance and implementation complexity, we assume that merely two users can be allocated and multiplexed on the same SC in each BS.

Due to $(C 1),(C 2)$, and the non-convex constraint in $(C 4)$, it is challenging to obtain the global optimal solution in polynomial time. Hence, we divide (8) into three subproblems, namely user association, subchannel assignment and power allocation, to achieve an effective near-optimal solution.

\section{AdAptive FuZzy Logic BASEd MUlti-Criterion USER ASSOCIATION}

At present, two kinds of user association methods are mostly mentioned in literatures: distance based [9] [11] and channel gain based method [10] [12], which are expressed as if $d_{l}>d_{\text {pre }}$, user $l$ is in the coordinated mode,

if $H_{l}<H_{\text {pre }}$, user $l$ is in the coordinated mode, where $d_{\text {pre }}$ and $H_{\text {pre }}$ are predetermined thresholds.

The other effective scheme is to perform a ranking based user association. Based on a ranking criterion, users are sorted

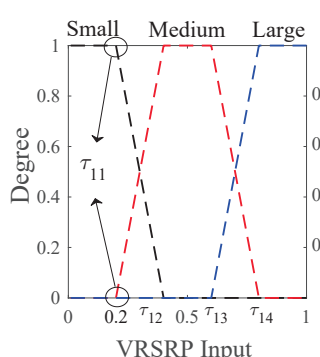

VRSRP Input
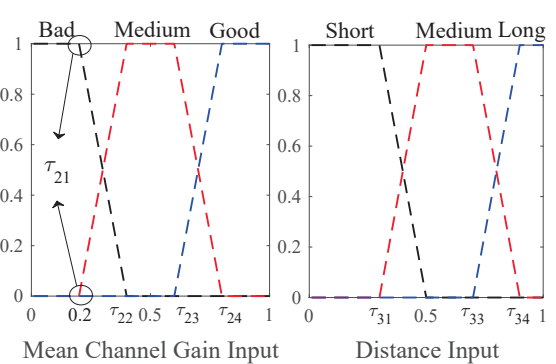

Fig. 2. Fuzzy membership function of three normalized inputs.

in order and some top users are chosen as the coordinated users. However, the performance of the distance or channel gain based user association method is severely affected by fading, shadowing, and inter-cell interference. As a result, some users' coordination mode cannot be appropriately selected, which decreases the system performance. Therefore, we consider multiple criteria in user association and introduce FL to make a balance among them.

1) Fuzzy Inputs and Output: For each user, the distance, mean channel gain and variance of RSRP (VRSRP) are three inputs. Generally, if the RSRPs are close to each other, the user is more likely in an area with stronger inter-cell interference and has a higher probability to be chosen in the coordinated mode. The FL output denotes users' respective suitability for coordinated mode considering three inputs.

$X=d \in\{$ Short, Medium, Long $\}$, $Y=\bar{H} \in\{$ Bad, Medium, Good $\}$, $Z=\operatorname{VRSRP} \in\{$ Small, Medium, Large $\}$, $O=$ Output $\in\{$ Very Bad, Bad, Medium, Good, Very Good $\}$.

2) Fuzzification and Defuzzification Process: In order to perform the fuzzificaiton process, three inputs and one output should be mapped to fuzzy sets.

Fig. 2 presents the FL membership function of normalized inputs. Since all three inputs change linearly and continuously, Trapezoidal function is chosen to generate membership function [13]. For one input, membership function generates one membership degree which is between 0 to 1 , according to the type of input. As there is no experience information of VRSRP and mean channel gain, similar to [7], their memberships initially have balanced distribution of three levels (i.e., $\tau_{11}=0.2, \tau_{12}=0.4, \tau_{13}=0.6, \tau_{14}=0.8$ ). Also, as an empirical parameter, the distribution of levels of distance skews to right (i.e., $\tau_{31}=0.3, \tau_{33}=0.7, \tau_{34}=0.9$ ), which ensures that fewer center users are switched to coordinated mode.

However, the aforemetioned fuzzy membership function is predetermined and cannot be tuned adaptively to the change of network environment, which results in performance degradation. Hence, in order to make the FL based scheme adaptive to different situations and enlarge the difference of suitability of the users under deep shadowing and fading, we set $\tau_{11}$ and $\tau_{21}$ as $\tau_{11}=0.2[1-(\theta+1) /(10 \gamma)], \tau_{21}=0.2[1-(\theta+1) /(10 \gamma)]$, with path loss exponent $\gamma$ and shadowing standard deviation $\theta$. 
The membership degree of the FL output set is given by

$$
\lambda_{o} \triangleq \min \left(\lambda_{X}, \lambda_{Y}, \lambda_{Z}\right)
$$

where $\lambda_{X}, \lambda_{Y}$ and $\lambda_{Z}$ denote the degree of membership of distance, mean channel gain and VRSRP, respectively. Then we transform the output set $\lambda_{o}$ into a crisp number $\Gamma$, which is between $[0,1]$ :

$$
\Gamma=\frac{\sum\left(\lambda_{o} \cdot O_{\text {mid }}\left(\lambda_{o}\right)\right)}{\sum \lambda_{o}},
$$

where $O_{\text {mid }}\left(\lambda_{o}\right)$ denotes the middle value of the normalized numerical value of $\lambda_{o}$. The process of fuzzification and defuzzification is presented in [7] and [13]. Due to space limitations, the detailed process is omitted here.

After the FL output crisp number of all $L$ users have been obtained, we rank the users in descending order based on $\Gamma$ and choose the top $L_{C}$ users to work in the coordinated mode.

\section{SCG BASED JOINT RESOURCE ALLOCATION}

As mentioned above, to strike an attractive balance between the performance and complexity of SIC, we consider only two users can be simultaneously multiplexed on the same SC for each BS. Following [2], we consider the number of users served by each $\mathrm{BS} b$ is $L_{b}=2 M$, and the number of users selected to work in the coordinated mode is $L_{C}=M$. For each BS, since the coordinated users usually suffer from poor channel conditions, they are regarded as weak users, while the noncoordinated users are taken as strong users.

For a non-coordinated user $l$ allocated to SC $m$, its SCG is $H_{B_{l}, l, m}$. According to (2), the performance of non-coordinated user $l$ gets better as its SCG increases. Nevertheless, the increasing SCG of user $l$ inevitably has effect on the intracell and inter-cell interference of other users on the same SC, as well as the power allocation among users. Unfortunately, there is little analysis at present to theoretically explore the effect of the increasing SCGs of non-coordinated users on the variation of transmission power in coordinated NOMA.

Theorem 1: In a downlink multi-cell coordinated NOMA system, the total transmission power $P_{t}$ is mono-decreasing with respect to the SCGs of non-coordinated users.

Proof of Theorem 1: See Appendix.

Lemma 1: For the subchannel allocation in coordinated NOMA, the selection of a non-coordinated user with higher SCG leads to less transmission power consumption than any other non-coordinated users with lower SCG.

1) Algorithm Description: We first perform the subchannel allocation for the non-coordinated users in each cell separately. Denote the number of non-coordinated users served by BS $b$ as $L_{M_{b}}=M$. First, for each SC $m$, we rank the $M$ noncoordinated users in descending order based on their SCGs and form a $M \times 1$ candidate list $\Lambda_{b, m}$. After that, we allocate SCs based on the candidate lists. For each SC $m$, it chooses the first user (e.g., user $l$ ) in $\Lambda_{b, m}$ as its candidate user if it has not been selected. Otherwise, according to Theorem 1 , user $l$ selects the SC with higher SCG and rejects the other one. After that, user $l$ is removed from the candidate list of the rejected
SC. Repeat these steps until all $M$ SCs and $M$ non-coordinated users are allocated.

After that, we form the candidate list $\Lambda_{C, m}$ of each SC $m$ for coordinated users by ranking them in descending order based on their SCGs. Then, a joint subchannel and power allocation is presented. For each SC $m$, it initially chooses the first user (e.g., user $l$ ) in $\Lambda_{C, m}$ if user $l$ has not been assigned. Since the noncoordinated users have been assigned, we find the transmission power allocation $P_{t, m}^{*}$ on SC $m$ by exhaustive search or using (14), and store $P_{t, m}^{*}$ as the candidate transmission power of SC $m$. Otherwise, assume user $l$ has been assigned to SC $n$, it finds the transmission power on SC $m P_{t, m}^{*}$, then compares $P_{t, m}^{*}$ with $P_{t, n}^{*}$ and selects the $\mathrm{SC}$ with lower transmission power. After that, user $l$ is removed from the candidate list of the rejected SC. Repeat these steps until all of the $M$ SCs and $L_{C}$ coordinated users are assigned. The procedures are described in Algorithm 1.

2) Closed-Form Optimal Solution to Power Allocation: For multi-cell coordinated NOMA, assume the non-coordinated user on SC $m$ in cell $l(l=1, \ldots, B)$ is $l$ and the coordinated user is $(B+1)$. Define the transmission power matrix as $\mathbf{P}=\left[\mathbf{P}_{1} \ldots \mathbf{P}_{M}\right]^{T}$, with $\mathbf{P}_{m}=\left[p_{1, m} \ldots p_{b, m} \ldots p_{B+1, m}\right]^{T}(m=$ $1, \ldots, M)$. Note that constraint $(C 4)$ in $(8)$ can be rewritten as

$$
\begin{gathered}
(\widetilde{C 4}): p_{l, m} \sum_{b \in B_{l}} H_{b, l, m}+\beta \sum_{b \in B_{l}} H_{b, l, m} \sum_{i \in\left\{U_{m, b}, l\right.} p_{i, m}+ \\
\beta \sum_{b \in\left\{B \backslash B_{l}\right\}} H_{b, l, m} \sum_{j \in\left\{U_{m}, b\right\}} p_{j, m}+\beta \sigma_{m}^{2} \geq 0,
\end{gathered}
$$

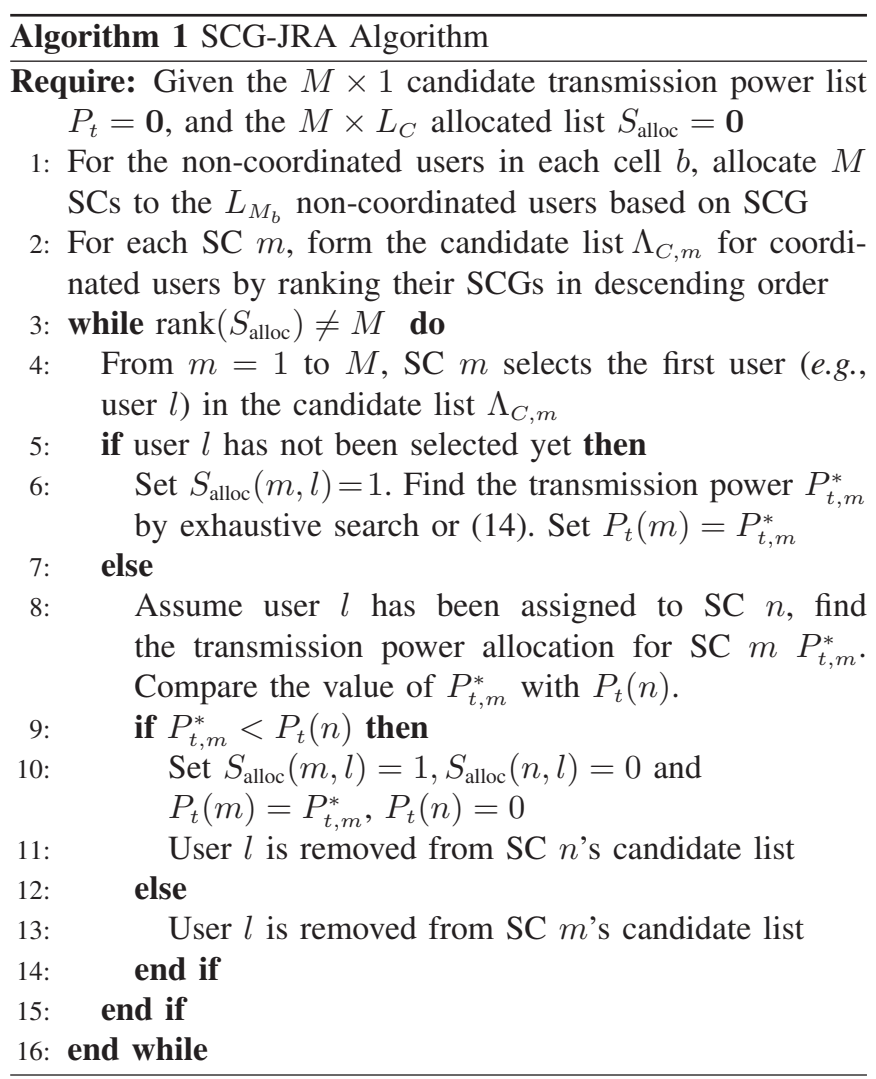




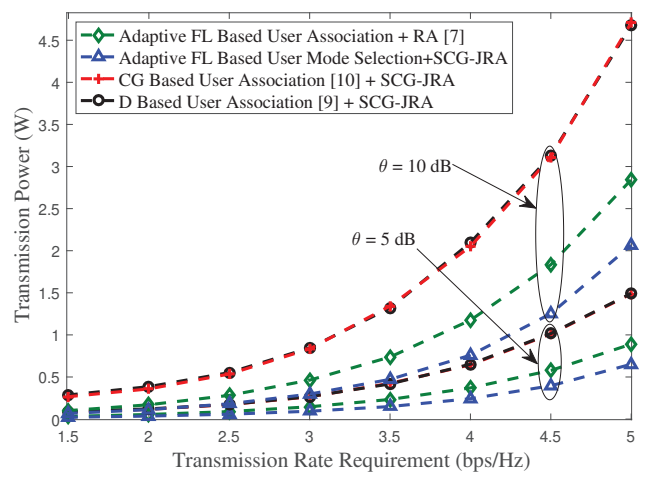

Fig. 3. Transmission power consumption performance for green coordinated NOMA systems under QoS requirement.

where $\beta=1-2^{r_{\min }}$. Note that $(\widetilde{C 4})$ can be further rewritten in linear matrix form as

$$
\left[\beta H_{1, l, m} \ldots H_{l, l, m} \ldots \beta H_{B, l, m} \beta \sum_{i=1, i \neq l}^{B} H_{i, l, m}\right] \mathbf{P}+\beta \sigma_{m}^{2} \geq 0,
$$

which can be easily proved to be convex with respect to $\mathbf{P}$ [11] [12]. Hence, (8) can be reformulated as

$$
\underset{\mathbf{P}}{\arg \min } \eta(\mathbf{P})=P_{t},
$$

subject to $(\widetilde{C 4})$ and $(C 5)$. The Lagrange function for SC $m$ is

$$
\begin{aligned}
& L\left(\mathbf{P}_{m}, \mathbf{a}, \mu\right)= \\
& \sum_{b=1}^{B} \sum_{l \in U_{m}, b} p_{l, m}+\sum_{l=1}^{B+1} a_{l}\left(r_{\min }-r_{l}\right)-\sum_{l=1}^{B+1} \mu_{l} p_{l, m},
\end{aligned}
$$

where $a_{l}$ and $\mu_{l}$ are Lagrange multipliers. According to KarushKuhn-Tucker conditions, it is easy to prove $\mu_{l}=0, a_{l}>0$. Differentiating $L\left(\mathbf{P}_{m}, \mathbf{a}, \mu\right)$ with respect to $a_{l}(l=1, \ldots, B+1)$ and equaling them to zero, we have

$$
\begin{aligned}
& {\left[\begin{array}{cccccc}
H_{1,1, m} & \ldots & \beta H_{b, 1, m} & \ldots & \beta H_{B, 1, m} & \beta \sum_{i=2}^{B} H_{i, 1, m} \\
\ldots & & \ldots & & \\
\beta H_{1, B, m} & \ldots & \beta H_{b, B, m} & \ldots & H_{B, B, m} & \beta \sum_{i=1}^{B-1} H_{i, B, m} \\
\beta H_{1, B+1, m} \ldots & \beta H_{b, B+1, m} \ldots & \beta H_{B, B+1, m} & \sum_{i=1}^{B} H_{i, B+1, m}
\end{array}\right]\left[\begin{array}{c}
P_{1, m} \\
\ldots \\
P_{B, m} \\
P_{B+1, m}
\end{array}\right]} \\
& -\left[\left(-\beta \sigma_{m}^{2}\right) \ldots\left(-\beta \sigma_{m}^{2}\right)\right]_{1 \times(B+1)}^{T}=\mathbf{A}_{m} \mathbf{P}_{m}-\mathbf{Q}=\mathbf{0},
\end{aligned}
$$

where $\mathbf{A}_{m}$ is an invertible square matrix.

As a result, the solution of $\mathbf{P}_{m}$ can be obtained as

$$
\mathbf{P}_{m}^{*}=\left[\begin{array}{lllll}
p_{1, m}^{*} & \cdots & p_{b, m}^{*} & \cdots & p_{B+1, m}^{*}
\end{array}\right]^{T}=\mathbf{A}_{m}{ }^{-1} \mathbf{Q} .
$$

\section{NuMERICAL RESUlts}

In this section, numerical results are provided to demonstrate the performance of our proposed adaptive FL based multicriterion user association and SCG-JRA algorithms for green coordinated NOMA. The number of cells is 2 and the radius for

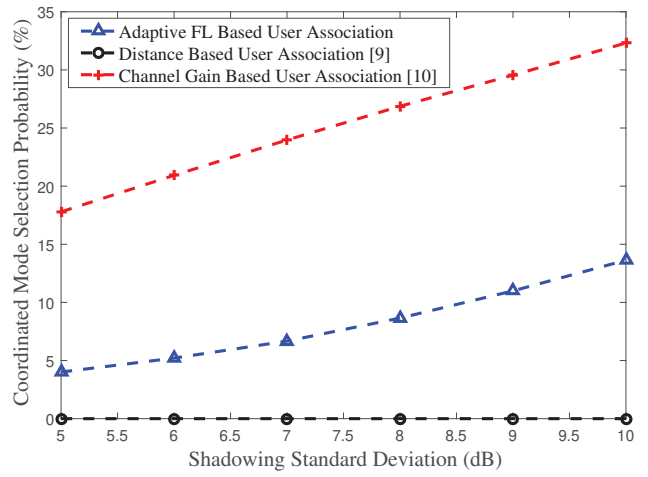

Fig. 4. Coordinated mode selection probability of cell center users under deep shadowing in multi-cell coordinated NOMA.

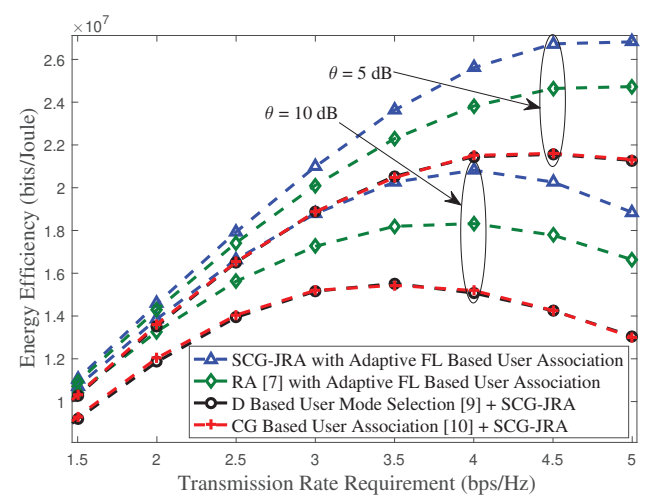

Fig. 5. EE performance for green coordinated NOMA systems.

each cell is $500 \mathrm{~m}$. The bandwidth is $W=5 \mathrm{MHz}$, and the variance of additional white Gaussion noise is $\sigma_{m}^{2}=-112 \mathrm{dBm}$. Subchannels are modeled as independent Rayleigh fading, and the path loss model is given by $P L(d)=128.1+37.6 \log _{10}(d)$ [14]. We assume a total of $L=9$ users as illustrated in Fig. 1 and the circuit power is set to $30 \mathrm{dBm}$ [9].

As can be seen from Fig. 3, the transmission power performance of the proposed SCG-JRA algorithm with adaptive FL based user association is substantially better than that of the single-criterion based RA schemes. For example, when shadowing standard deviation is $\theta=10 \mathrm{~dB}$ and $r_{\min }=4.5$ bps/Hz, the SCG-JRA algorithm with adaptive FL based user association requires $59.7 \%$ less transmission power than the RA schemes assisted by single-criterion user association [9] [10]. It also outperforms the RA scheme in [7] under the same user association result. Furthermore, the performance gap becomes larger as $\theta$ increases. The reason is that the adaptive FL based scheme has considered three parameters and tunes its fuzzy membership function adaptively to the change of network environment, which is more effective against fading, shadowing and multi-cell interference than the single-criterion based user association methods. This can also be proven by Fig. 4 , which shows the coordinated mode selection probability of cell center users under deep shadowing. Our adaptive FL based 


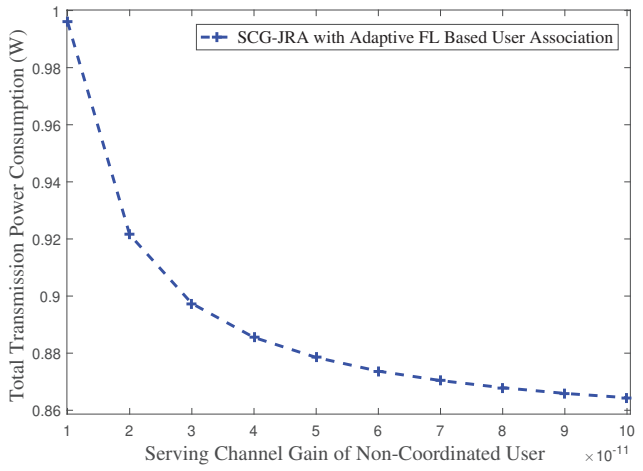

Fig. 6. Illustration of Theorem 1: monotone decreasing of total transmission power with respect to the serving channel gain of non-coordinated user.

user association makes a balance among multiple performance limiting factors and therefore is more reliable.

In Fig. 5, the SCG-JRA with adaptive FL based user association is much more energy-efficient than the single-criterion based methods [9] [10] and the RA scheme in [7]. When $\theta=10 \mathrm{~dB}$ and $r_{\min }=4 \mathrm{bps} / \mathrm{Hz}$, the EE achieved by SCGJRA with adaptive FL based user association is $38.1 \%$ higher than that achieved by the RA schemes with single-criterion user association [9] [10], and $13.6 \%$ higher than that achieved by the RA scheme in [7] under the same user association result.

Fig. 6 shows the mono-decreasing of transmission power with respect to the SCG of a randomly selected noncoordinated user with $L=3$, and $r_{\min }=4 \mathrm{bps} / \mathrm{Hz}$, which is consistent with Theorem 1.

\section{CONCLUSIONS}

We have proposed a novel joint RA algorithm, referred to as SCG-JRA, as well as an adaptive FL based multi-criterion user association algorithm, for multi-cell coordinated NOMA systems. The proposed system achieves a total transmission power reduction of up to $59.7 \%$ and an EE enhancement of up to $38.1 \%$ over the previous RA schemes assisted by singlecriterion based user association [9][10], thanks to the effectiveness of joint RA and the robustness of adaptive FL based user association against the combined effect of shadowing, fading and inter-cell interference.

APPENDIX:

\section{PROOF OF THEOREM 1}

Based on (7) and (14), taking the partial derivative of total transmission power with respect to $H_{l, l, m}$ yields

$$
\frac{\partial P_{t}}{\partial H_{l, l, m}}=\sum \frac{\partial \mathbf{P}_{m}}{\partial H_{l, l, m}}=\sum-\mathbf{A}_{m}^{-1} \frac{\partial \mathbf{A}_{m}}{\partial H_{l, l, m}} \mathbf{A}_{m}^{-1} \mathbf{Q} \text {. }
$$

Denote the inverse of $\mathbf{A}_{m}$ as $\mathbf{A}_{m}{ }^{-1}=\frac{\mathbf{D}}{\left|\mathbf{A}_{m}\right|}$, with $\mathbf{D}$ as the adjoint matrix and $\left|\mathbf{A}_{m}\right|$ denoting the determinant of $\mathbf{A}_{m}$, respectively. Then (15) can be reformulated as

$$
\sum \frac{\partial \mathbf{P}_{m}}{\partial H_{l, l, m}}=\frac{\left(\sum_{b=1}^{B+1} D_{l, b}\right)\left(\sum_{b=1}^{B+1} D_{b, l}\right)}{\left|\mathbf{A}_{m}\right|^{2}}\left(\beta \sigma_{m}^{2}\right),
$$

where $D_{l, b}$ denotes the element on the $l$-th row, $b$-th column of $\mathbf{D}, \beta=1-2^{r_{\min }}<0$. Notice that the diagonal elements in $\mathbf{A}_{m}$ are the SCG of users, while the non-diagonal elements are the product of users' interference and $\beta$. Based on the unique structure of $\mathbf{A}_{m}$, we obtain

$$
\sum_{b=1}^{B+1} D_{l, b}=\left(F_{l}-\sum_{i=2}^{B}(-\beta)^{i} F_{l, i}\right)+\sum_{b=1, b \neq l}^{B+1} \sum_{i=1}^{B}(-\beta)^{i} G_{b, i},
$$

where $F_{l}$ denotes the product of users' SCG except user $l$, the positive terms $F_{l, i}$ and $G_{b, i}$ stand for the products of users' SCG and interference on SC $m$. Note that for non-coordinated user $l$, the SCG is generally much larger than the channel gain from other BSs, as a result, we have $F_{l}>>F_{l, i}, i=2, \ldots, B$, which implies $\sum_{b=1}^{B+1} D_{l, b}>0$. We can also prove $\sum_{b=1}^{B+1} D_{b, l}>0$ by utilizing the similar methodology. Hence, we conclude

$$
\frac{\partial P_{t}}{\partial H_{l, l, m}}=\sum \frac{\partial \mathbf{P}_{m}}{\partial H_{l, l, m}}<0
$$$$
\text { REFERENCES }
$$

[1] Z. Wei, X. Zhu, S. Sun, J. Wang, and L. Hanzo, "Energy efficient fullduplex cooperative non-orthogonal multiple access," IEEE Transactions on Vehicular Technology, vol. 67, no. 10, pp. 10123-10128, Aug. 2018.

[2] F. Fang, H. Zhang, J. Cheng, and V. C. M. Leung, "Energy-efficient resource allocation for downlink non-orthogonal multiple access network," IEEE Trans. Commun., vol. 64, no. 9, pp. 3722-3732, Jul. 2016.

[3] X. Sun, N. Yang, S. Yan, Z. Ding, D. W. K. Ng, C. Shen, and Z. Zhong, "Joint beamforming and power allocation in downlink NOMA multiuser MIMO networks," IEEE Trans. Wireless Commun., vol. 17, no. 8, pp. 5367-5381, Aug. 2018

[4] Y. Jiang, X Zhu, E. Lim, Y Huang, and H. Lin, "Low-complexity semiblind multi-CFO estimation and ICA-based equalization for CoMP OFDM systems," IEEE Transactions on Vehicular Technology, vol. 63 , no. 4, pp. 1928-1934, Oct. 2014.

[5] M. S. Ali, E. Hossain, and D. I. Kim, "Coordinated multipoint transmission in downlink multi-cell NOMA systems: models and spectral efficiency performance," IEEE Wireless Commun., vol. 25, no. 2, pp. 24-31, Apr. 2018.

[6] Z. Zhang, G. Yang, Z. Ma, M. Xiao, Z. Ding, and P. Fan, "Heterogeneous ultradense networks with NOMA system architecture: coordination framework and performance evaluation," IEEE Veh. Technol. Mag., vol. 13, no. 2, pp. 110-120, Jun. 2018.

[7] H. Zeng, X. Zhu, Y. Jiang, Y. Zuo, Z. Wei and T. Wang, "Fuzzy Logic Based Multi-Criterion User Selection and Resource Allocation for Green Coordinated NOMA," in Proc. IEEE Global Commun. Conf. (GLOBECOM'18), Abu Dhabi, UAE, Dec. 2018.

[8] A. Beylerian and T. Ohtsuki, "Coordinated non-orthogonal mulitple access (CO-NOMA)," in Proc. IEEE Global Commun. Conf. (GLOBECOM'16) Wksp., Washington DC, USA, Dec. 2016.

[9] Z. Liu, G. Kang, L. Lei, N. Zhang, and S. Zhang, "Power allocation for energy efficiency maximization in downlink CoMP systems with NOMA," in Proc. IEEE Wireless Commun. and Networking Conf. (WCNC'17), San Francisco, CA, USA, May 2017.

[10] Y. Tian, A. Nix, and M. Beach, "On the performance of opportunistic NOMA in downlink CoMP networks," IEEE Commun. Letts., vol. 20, no. 5, pp. 998-1001, Mar. 2016.

[11] J. Lee, Y. Kim, H. Lee, B. Ng, D. Mazzarese, J. Liu, W. Xiao, and Y. Zhou, "Coordinated multipoint transmission and reception in LTEAdvanced systems," IEEE Commun. Mag., vol. 50, no. 11, pp. 44-50, Nov. 2012.

[12] Q. Zhang and C. Yang, "Transmission mode selection for downlink coordinated multipoint systems," IEEE Trans. Veh. Technol., vol. 62, no. 1 , pp. 456-471, Sep. 2013.

[13] T. J. Ross, Fuzzy Logic with Engineering Applications. John Wiley and Sons, Chichester, 2004.

[14] Further Advancements for E-UTRA Physical Layer Aspects. 3GPP TR 36.814 V9.0.0, Mar. 2010. Available: http://www.3gpp.org/ftp/Specs/archive/36_series/36.814/ 